\title{
The early B-type star Rho Ophiuchi A is an X-ray lighthouse
}

\author{
Ignazio Pillitteri ${ }^{1,2}$, Scott J. Wolk ${ }^{2}$, Fabio Reale ${ }^{1}$, and Lida Oskinova ${ }^{3}$ \\ 1 INAF-Ossevatorio Astronomico Palermo, Piazza del Parlamento 1, 90134 Palermo, Italy \\ e-mail: pilli@astropa.inaf.it \\ 2 Harvard-Smithsonian CfA, 60 Garden st, Cambridge, MA 02138, USA \\ 3 Institut für Physik und Astronomie, Universität Potsdam, USA Karl-Liebknecht-Strasse 24/25, 14476 Potsdam-Golm, Germany
}

Received 15 November 2016 / Accepted 20 March 2017

\begin{abstract}
We present the results of a $140 \mathrm{ks} X M M$-Newton observation of the $\mathrm{B} 2$ star $\rho$ Oph A. The star has exhibited strong X-ray variability: a cusp-shaped increase of rate, similar to that which we partially observed in 2013, and a bright flare. These events are separated in time by about $104 \mathrm{ks}$, which likely correspond to the rotational period of the star (1.2 days). Time resolved spectroscopy of the X-ray spectra shows that the first event is caused by an increase of the plasma emission measure, while the second increase of rate is a major flare with temperatures in excess of $60 \mathrm{MK}(k T \sim 5 \mathrm{keV})$. From the analysis of its rise, we infer a magnetic field of $\geq 300 \mathrm{G}$ and a size of the flaring region of $\sim 1.4-1.9 \times 10^{11} \mathrm{~cm}$, which corresponds to $\sim 25 \%-30 \%$ of the stellar radius. We speculate that either an intrinsic magnetism that produces a hot spot on its surface or an unknown low mass companion are the source of such X-rays and variability. A hot spot of magnetic origin should be a stable structure over a time span of $\geq 2.5 \mathrm{yr}$, and suggests an overall large scale dipolar magnetic field that produces an extended feature on the stellar surface. In the second scenario, a low mass unknown companion is the emitter of X-rays and it should orbit extremely close to the surface of the primary in a locked spin-orbit configuration, almost on the verge of collapsing onto the primary. As such, the X-ray activity of the secondary star would be enhanced by its young age, and the tight orbit as in RS Cvn systems. In both cases $\rho$ Oph would constitute an extreme system that is worthy of further investigation.
\end{abstract}

Key words. stars: activity - stars: individual: Rho Ophiuchi - stars: early-type - stars: magnetic field - starspots - X-rays: stars

\section{Introduction}

The emission of X-rays from massive stars is explained as due to strong stellar winds and shocks in $\mathrm{O}$ stars through a mechanism of line deshadowing instability (LDI; Feldmeier et al. 1997a,b; Owocki et al. 1998). In the case of binarity, wind-wind collision gives an additional source of X-rays, sometimes coupled with the presence of magnetic fields that drive ionized winds and increase shock temperatures (Babel \& Montmerle 1997; ud-Doula \& Owocki 2002). The production of X-rays by stellar winds appears less realistic in B-type stars because of their weaker winds with respect to those from $\mathrm{O}$ and WR stars.

Moving from $\mathrm{O}$ to early B-type stars, the rate of detection in X-rays among B stars falls to about $50 \%$, where hard X-ray emission in a few cases are a signature of the presence of strong magnetic fields or due to an unknown, low mass young and active companion. X-rays from single early B stars are observed in a few cases, and their origin is likely linked to their strong magnetism. Cases of spots in young stars of NGC 2264, which presumably have a magnetic origin, are given by Fossati et al. (2014). Recent spectroscopic surveys of O-B stars have revealed that about $7 \%$ of these stars are magnetic (Wade et al. 2014; Fossati et al. 2015). In a few cases magnetic fields of strength of a few $\mathrm{kG}$ are measured, likely accompanied by peculiar photospheric abundances.

$\rho$ Oph is a multiple system of B-type stars. They are part of one of the closest and densest sites of active star formation. In particular $\rho$ Oph $\mathrm{A}+\mathrm{B}$ is a binary system of two B2 stars separated by about $310 \mathrm{AU}$ at a distance of $111 \pm 10 \mathrm{pc}$ from the Sun (parallax $9 \pm 0.9$ mas, separation $\sim 2.8^{\prime \prime}$; van Leeuwen 2007; Malkov et al. 2012), the orbital period of the system is about $2400 \pm 330 \mathrm{yr}$ (Malkov et al. 2012). $\rho$ Oph A rotates with $v \sim 300 \mathrm{~km} \mathrm{~s}^{-1}$ (van Belle 2012; Glebocki \& Gnacinski 2005; Uesugi \& Fukuda 1982), has a mass of about 8-9 $M_{\odot}$, and radius of $\sim 8 R_{\odot}$.

In 2013 we observed $\rho$ Oph with XMM-Newton discovering that it emits X-rays. In particular, we observed a significant rise of X-ray flux in the last 20-25 ks of the exposure, accompanied by plasma temperatures around $\sim 3 \mathrm{keV}$ (Pillitteri et al. 2014, hereafter Paper I). We hypothesized that either an active spot was emerging on the stellar surface, or an unknown low mass companion was causing the feature. Along with $\rho$ Oph, we discovered a young cluster of about 25 pre-main sequence (PMS) stars, which are mostly without circumstellar disks and of 5-10 Myr in age. These stars were likely born together with the $\rho$ Oph stars during the first event of star formation in the cloud (Pillitteri et al. 2016).

In this paper we present the results of a follow-up observation of $\rho$ Oph with XMM-Newton, with a duration of $140 \mathrm{ks}$. The aim of the observation was to monitor the X-ray emission of $\rho$ Oph for a full rotational period of the star $(\sim 1.2$ days or $104 \mathrm{ks}$ ) and understand the origin of its X-rays. The structure of the paper is the following: in Sect. 2 we describe the observations and data analysis; Sect. 3 present the results; and in Sect. 5 we discuss them and present our conclusions.

\section{Observations and data analysis}

XMM-Newton observed $\rho$ Oph on February 222016 for $140 \mathrm{ks}$ (ObsId 0760900101). We used EPIC camera as the first instrument with the Thick filter to prevent UV leakage due to the brightness of the target $(U=4.30)$. For the same reason, OM 


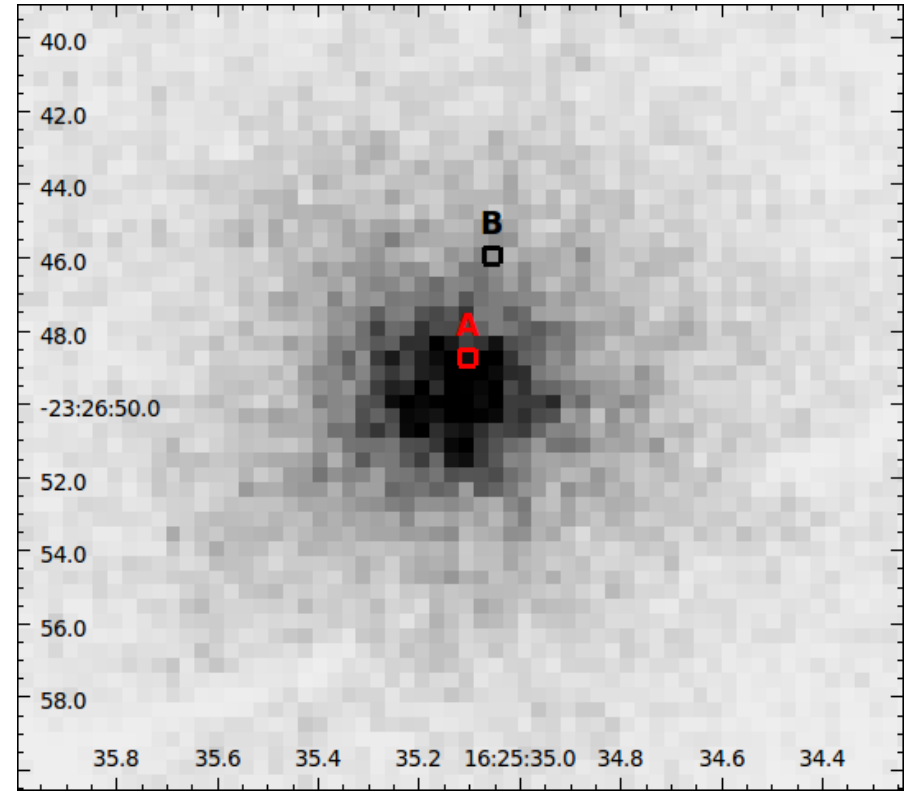

Fig. 1. MOS 1 image of $\rho$ Oph, the positions of the two components from SIMBAD database are indicated with colored boxes. The centroid of X-ray events is much closer to the A component. The scale is linear, the events have been rebinned in blocks of $0.4^{\prime \prime}$ and smoothed with a Gaussian of $\sigma=1$ pixel. The separation between the two components is $\sim 2.8^{\prime \prime}$. The core of the point spread function of MOS1 is about $2^{\prime \prime}$ and the astrometric precision is $\sim 1.2^{\prime \prime}$.

exposures were not taken for the safety of the instrument. The observation data files (ODFs) were reduced with SAS ver. 15.0 to obtain tables of events for MOS 1, 2 and $p n$ filtered in $0.3-$ $8.0 \mathrm{keV}$, and with filters FLAG $=0$ and PATTERN $<=12$ as prescribed by the SAS guide. We used a circular region of radius $35^{\prime \prime}$ for both source and background events to select the events of $\rho$ Oph. For $p n$ we used a background extracted from a region at the same distance from the readout node of the same chip of the source, as prescribed by the SAS guide book. In Paper I we associated the X-ray emission with the A component of $\rho \mathrm{Oph}$; the MOS1 image in Fig. 1 shows that this is a reasonable assumption, given that the centroid of the X-ray events is much closer to the position of $\rho$ Oph A and completely offset with respect to the position of $\rho$ Oph B.

Spectra, response matrices ( $\mathrm{rmf}$ ), ancillary files (arf), and light curves of $\rho$ Oph A were obtained with the specific SAS tasks (evselect, rmfgen, arfgen, backscale, and specgroup). The spectra were analyzed with XSPEC ver. 12.8.0, while generic software R language scripts and custom plotting routines were used to produce plots and calculate derived quantities of interest.

To understand the physical changes in the emitting plasma of $\rho$ Oph A, we divided the $p n$ light curve in intervals and accumulated the spectra from each time interval. We chose to obtain spectra with about 1600 counts each, which represents a trade off to preserve details of the temporal changes of the spectrum and its count statistics. In this way we divided the light curve in 37 time intervals of variable duration ( 1.2-6.0 ks, see Fig. 2). For each time bin we obtained $p n$ spectra and related calibration files ( $r m f$ and arf). We used a thermal model with absorption composed of two APEC components and a global PHABS photoelectric absorption $\left(N_{\mathrm{H}}\right)$. We kept $N_{\mathrm{H}}$ fixed to $3 \times 10^{21} \mathrm{~cm}^{-2}$ (see Paper I), formally $Z=0.3 Z_{\odot}$, while temperatures and normalization factors were left free to vary. Although the metallicity of Galactic B stars is well established (Nieva \& Przybilla 2012), sub-solar abundances reflect a peculiar behavior observed in active stellar coronae (see Sect. 3.2). XMM-Newton allows us to acquire simultaneously high resolution spectra of the central target with RGS gratings, provided it is bright enough. This was the case for $\rho$ Oph in the present $140 \mathrm{ks}$ exposure. The RGS spectra were obtained by first extracting the RGS1 and RGS2 spectra of $\rho$ Oph, then we added together the first order spectra with the SAS task rgscombine. We accumulated RGS spectra in several time intervals: full exposure (140 ks), first event (10-40 ks since start of exposure), second event (115-140 ks), the sum of first and second event (high state spectrum), and the relatively low activity interval in between (40-115 ks). For each time window we added together both RGS1 and RGS2 spectra of the first order. We focused on the range 5-20 $\AA$ because this is the range over which most of the flux and its changes are observed.

$\rho$ Oph has been observed with ESO-VLT and UVES spectrograph in 2001 and 2005 with different coverage of wavelengths (from $3000 \AA$ to $9000 \AA$ ), different exposure times (2s. to $15 \mathrm{~s}$.), and with a spectral resolution $R \sim 42000$. We used the reduced spectra from such observations to derive an estimate of the rotational velocity along the line-of-sight $v \sin i$ and to check the presence of lines from a low mass companion and any Doppler shift that could hint at the presence of such companion. We used a combination of Iraf/pyraf and a set of custom scripts to read the spectra in Iraf from the original MIDAS format, display the spectra, measure line absorption, remove the heliocentric component of Doppler shift due to the motion of the Earth, and calculate the cross-correlation function between spectra at two different epochs.

\section{Results}

Figure 2 shows the light curve of $\rho$ Oph A in the full band (0.3$8.0 \mathrm{keV})$, soft band (0.3-1.0 keV) and hard band (1.0-8.0 keV). During the observation $\rho$ Oph A exhibited two main episodes of variability: one at the beginning of the observation and another more powerful toward the end of the exposure. The first episode was a cusp-shaped increase of rate occurring during the first $40 \mathrm{ks}$. The peak of this event occurred at $t \sim 25 \mathrm{ks}$ and the decay phase appeared to be slightly steeper than the rise phase.

The second large increase of the rate occurred at $t \sim 116 \mathrm{ks}$ with a peak at around $t \sim 129 \mathrm{ks}$ and a decay that lasted until the end of the observation $(t \sim 139 \mathrm{ks})$. Likely, we missed the very end of this decay. Minor episodes of variability in the form of flares are visible at $t \sim 55 \mathrm{ks}$ and $105 \mathrm{ks}$. However, in the following we refer to the interval $40-110 \mathrm{ks}$ as the quiescent state, while the main two events of variability are referred to as the high state of $\rho$ Oph. The characteristics of the plasma and its changes in temperature and emission measure are discussed in detail in Sect. 3.2.

\subsection{Phased light curves and rotational velocity}

The rise of the rate in the first event is suspiciously similar to the rise of the rate observed in 2013 (Paper I), so we wonder whether the same mechanism is responsible for the increase of the rate observed twice in 2016. In particular, we hypothesize that a spot or an unseen companion transited during the observation, as speculated in Paper I. The time elapsed between the two main peaks of the light curve in Fig. 2 is approximately $104 \mathrm{ks}$ (or 1.2 days). If we consider this time as an initial guess for a phase-folding time, only a small adjustment of the period is 
I. Pillitteri et al.: The early B-type star Rho Ophiuchi A is an X-ray lighthouse

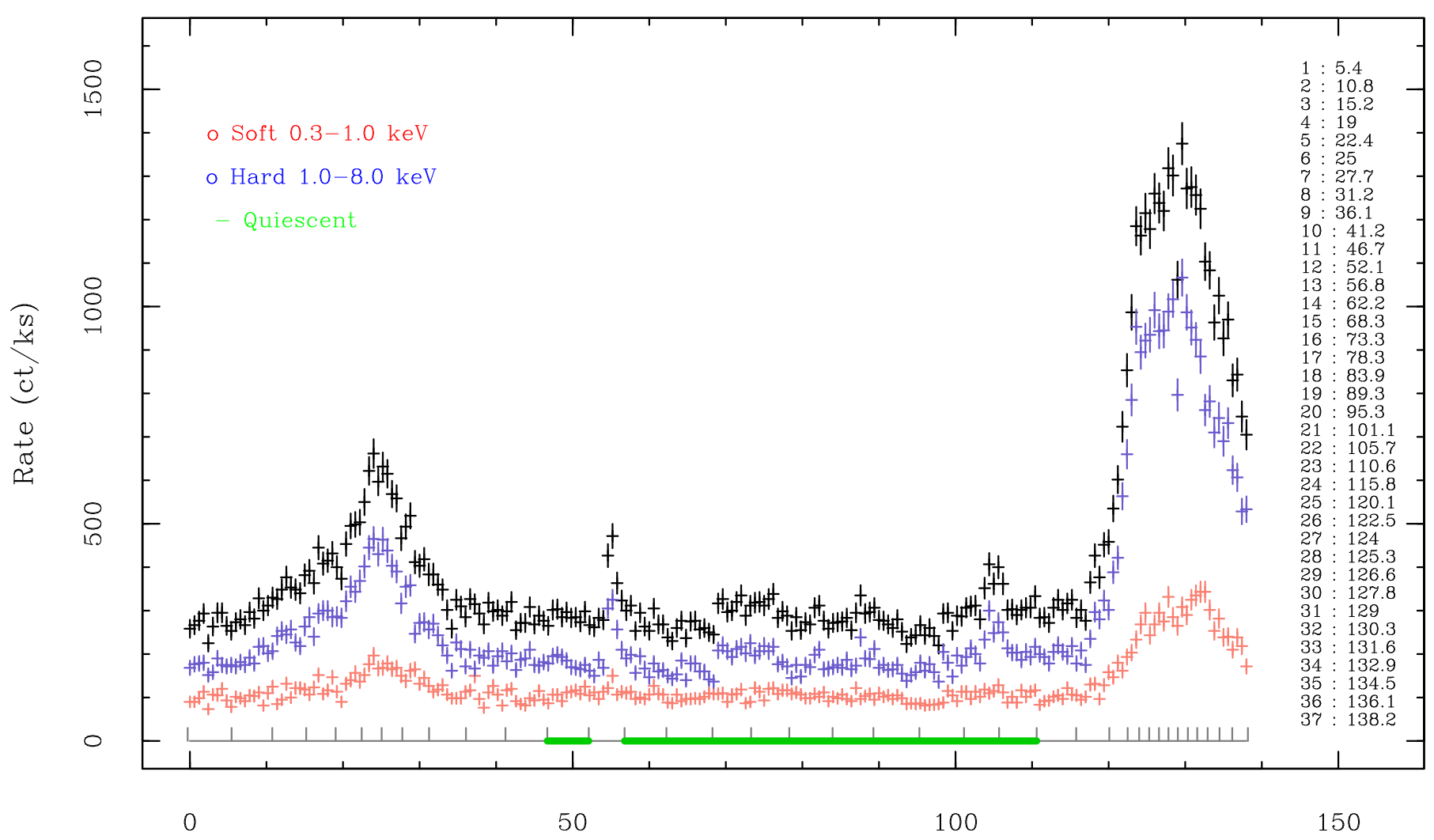

Time (ks)

Fig. 2. Plot of $p n$ light curve of $\rho$ Oph: full band ( $0.3-8.0 \mathrm{keV}$, black), soft band (0.3-1.0 keV, red), and hard band (1.0-8.0 keV, blue). Segments used in time resolved spectroscopy are indicated by ticks on the bottom scale and listed in the column to the right.

needed to aligning the rate peaks observed in 2013 and 2016, respectively. Figure 3 shows the phase folded light curves of 2013 and 2016, where as zero phase we used the beginning of the 2013 pn light curve. We thus find that a period of $104.11 \mathrm{ks}$ corresponding to 1.205 days aligns the three peaks, and this is our best estimate of the period of rotation of $\rho$ Oph A based on the periodic variations of its X-ray emission. This period corresponds to a rotational velocity of $\sim 340 \mathrm{~km} \mathrm{~s}^{-1}$ at the equator when assuming a stellar radius of $\sim 8 R_{\odot}$; the velocity derived from the 1.205 days period is roughly consistent with the rotational velocity of $\rho$ Oph A determined from optical observations (see van Belle 2012). The separation between the two epochs of observations is on the order of 1000 days and this leads to a precision of the period of $1 / 1000$ of day. Additional X-ray observations with shorter cadence could validate the measurement of the X-ray rotational period of $\rho$ Oph A.

We refined the estimate of $v \sin i$ of $\rho$ Oph A with an analysis of the Fourier transform of the line profile of $\mathrm{He}$ line at $6678 \AA$ from one of the available UVES spectra (Fig. 4, Gray 1992; Smith \& Gray 1976). The first minimum of the transform is related to the line broadening due to rotation and thus to $v \sin i$. The He line was chosen because it is well isolated from nearby lines, has a good signal, and offers an easy modeling of its profile. We used a window of wavelengths that encompasses the line (6671 $\AA-6685.5 \AA$ ), and we smoothed and normalized the profile with a lowess ${ }^{1}$ smoother. The Fourier transform was obtained on a sample of 5000 points interpolated along the line

\footnotetext{
1 As implemented in R, https://stat.ethz.ch/R-manual/ R-devel/library/stats/html/lowess.html
}

profile. The first minimum of the Fourier transform occurs at $\sigma=0.00275 \mathrm{c} / \AA$, which corresponds to $v \sin i=239.5 \mathrm{~km} \mathrm{~s}^{-1}$. The uncertainty is estimated at $\pm 10 \mathrm{~km} \mathrm{~s}^{-1}$. From $v \sin i$ and the rotational velocity, derived from the period obtained from the X-ray variability, we infer a $i$ angle between line of sight and rotational axis of $45 \pm 5^{\circ}$, taking into account an uncertainty of $0.5 R_{\odot}$ on the stellar radius. As first proposed in Paper I and discussed further in the next section, the presence of an active spot of magnetic origin and its periodic appearance would imply a magnetic field misaligned with the rotation axis.

On the other hand, if the X-rays were entirely produced by an active low mass companion, depending on the mass ratio, its motion around $\rho \mathrm{Oph}$ at a short distance would produce a wobble detectable as a Doppler shift in the spectra. For example, supposing a mass ratio of 1:15 (a late-K star), a period of 1.2 days, and an orbital velocity of the companion of 300 $350 \mathrm{~km} \mathrm{~s}^{-1}$ would produce a Doppler shift of $\sim 20 \mathrm{~km} \mathrm{~s}^{-1}$, which is easily detectable in the spectra of $\rho$ Oph. We first subtracted the component due to the motion of the Earth from the UVES spectra, then we cross-correlated the spectra taken at different epochs and of similar wavelength coverage (Tonry \& Davis 1979) avoiding regions with telluric lines. We did not detect any Doppler shift, meaning that either there is no companion or that the spectra were taken at approximately the same phase $\left(\mathrm{HJD}_{1}=2452\right.$ 132.6579; $\mathrm{HJD}_{2}=2453$ 618.4866, $\Delta \phi \sim 0.053$, for a period of 1.205 days). The phase difference implies a displacement of $19^{\circ}$ on the orbit. By supposing that $\rho$ Oph A has an average $v_{\text {orb }} \sim 20 \mathrm{~km} \mathrm{~s}^{-1}$, the $\sin i$ factor would amount to a difference of velocity of $6.5 \mathrm{~km} \mathrm{~s}^{-1}$ at the two phases; this 


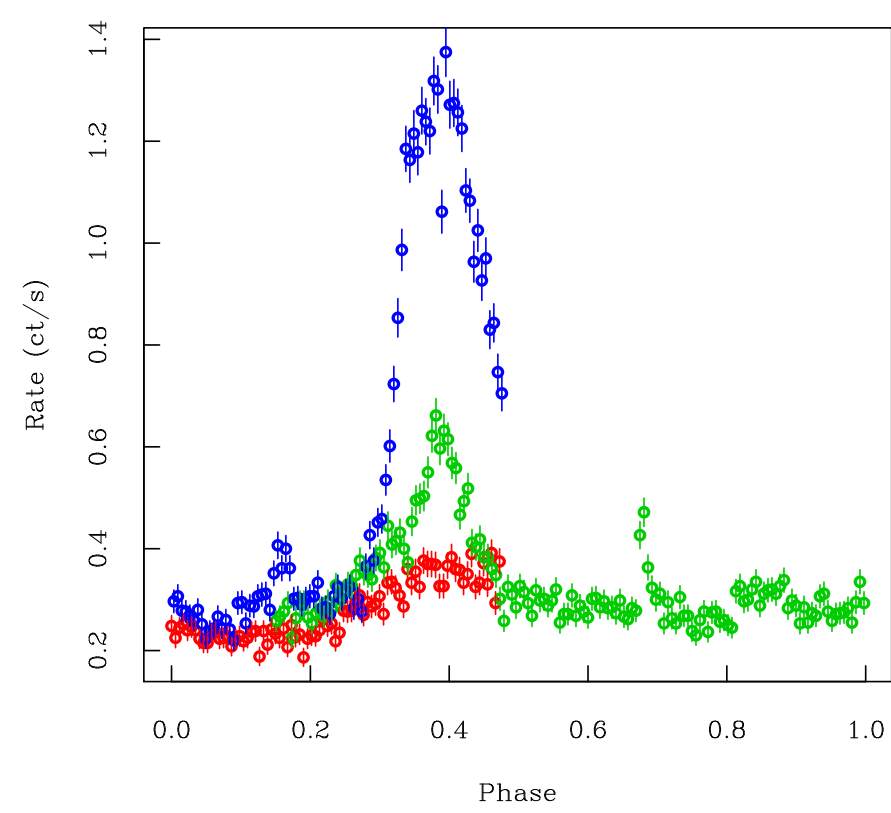

Fig. 3. Light curves of the rate phase-folded with a period of 1.205 days. The red dots indicate the 2013 observation, and the green and blue dots indicate the 2016 observation split in two periods. Phase zero was set arbitrarily to the beginning of 2013 observation. The three main peaks of X-ray emission can be put in phase with a period of 1.205 days, which corresponds to the rotational period of the star.
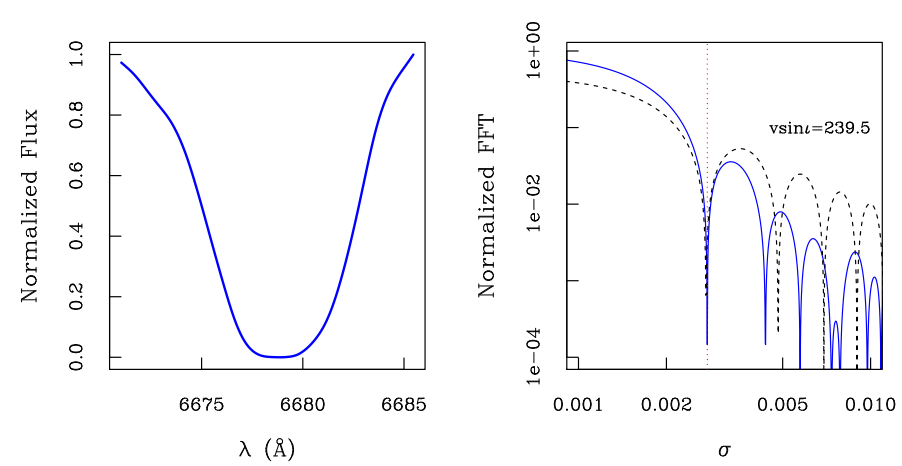

Fig. 4. Smoothed normalized profile of He line at $6678 \AA$ (left) and normalized amplitude of Fourier transform of the same profile (right, solid line). The dotted curve denotes the transform of the rotational broadening profile alone. The first minimum occurs at $\sigma \sim 0.00275 \mathrm{c} / \AA$ (vertical dotted line), corresponding to $v \sin i \sim 239.5 \mathrm{~km} \mathrm{~s}^{-1}$. Uncertainty on $v \sin i$ is estimated at around $\pm 10 \mathrm{~km} \mathrm{~s}^{-1}$.

difference is very similar to the UVES spectral resolution. A dedicated spectroscopic monitoring is thus required to detect a companion.

\subsection{Time resolved spectroscopy}

Here we discuss the spectral variations of $\rho$ Oph A with particular regard to the two main episodes of variability that characterized the X-ray light curve of $\rho$ Oph A. For each time interval, we performed a best fit of the spectrum with an absorbed thermal model composed of the sum of two APEC models. The free parameters were the temperatures and the respective emission measures of the thermal components, while absorption and abundances were kept fixed at $N_{\mathrm{H}}=3 \times 10^{21} \mathrm{~cm}^{-2}$ and $Z=0.3 Z_{\odot}$ in agreement with what was found in Paper I. By letting absorption vary, we obtained values of $N_{\mathrm{H}}$ consistent with the assumed value, and the variation of plasma temperatures were minimal

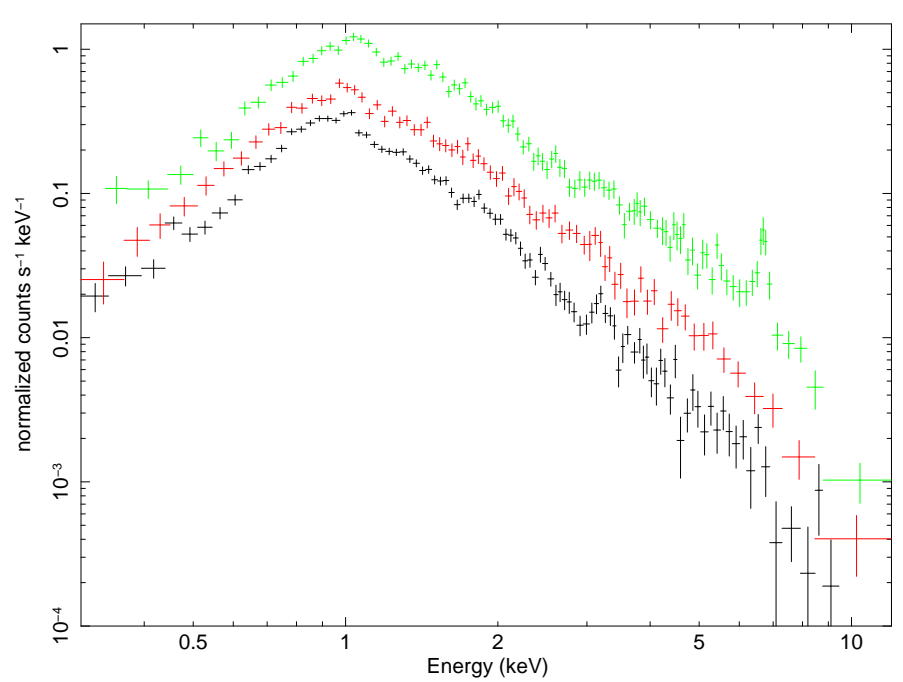

Fig. 5. Plot of $p n$ spectra of $\rho$ Oph A during quiescent phase (time bins $10-24$, bin 13 excluded, black spectrum), peak of the first event (bins 58, red spectrum), and peak of the second event (bins 27-33, green spectrum). This latter shows the complex of lines of ionized Fe at $6.7 \mathrm{keV}$ evidencing the high temperature of the plasma $(\geq 5.4 \mathrm{keV})$ reached during the peak of the second event.

with respect to the best fit with a fixed absorption. We discuss in detail the evolution of the hot component of the plasma, since the cool component had little variation during the observation, being comprised of $0.7 \mathrm{keV}<k T<1.2 \mathrm{keV}$ with a median of $k T \sim 0.9 \mathrm{keV}$ and standard deviation of $0.15 \mathrm{keV}$.

In Fig. 5 we show the $p n$ spectra of the quiescent phase at the peak of the two main events of variability. The spectrum changes significantly, especially during the second event when the temperature of the plasma was in excess of $5 \mathrm{keV}$. Evidence of such high temperatures is provided by the appearance of the complex of lines around $6.7 \mathrm{keV}$ owing to highly ionized Fe.

First event (intervals 1-10). The hot temperature during this event varied by $1.9 \mathrm{keV}<k T<2.5 \mathrm{keV}$ (Table A.1, Fig. 6) apart from interval number 6 when it had a sudden increase $(k T \sim 3.4 \mathrm{keV}$ ) of about $3 \sigma$ higher than the average of the previous values such heating rapidly vanished during the next interval (number 7). The spectrum corresponding to the intervals around the peak of the rate increase is shown in Fig. 5 (red curve). During intervals 8 through 10 the hot temperature slightly cooled down toward values lower than those seen at the beginning of the observation. We can conclude that, on average, only the plasma emission measure (EM) increased significantly during intervals 2-7, while the temperature was almost steady. This behavior fits the scenario in which a hot spot gradually appears on the stellar surface due to the stellar rotation $\left(P_{\text {rot }} \sim 1.205\right.$ days $\left.\sim 104.1 \mathrm{ks}\right)$. In interval number 6 either a short flare happened or the very hot core of the region was visible for a short time. The vanishing of the high rate is plausible with the gradual disappearance of the same spot at the opposite limb of the star.

Quiescent state. During intervals 11-24 the star showed a relatively small flare during interval number $13(\sim 55 \mathrm{ks}$ since the start of the observation, visible in four bins of the light curve of Fig. 2) and with a duration of approximately $5 \mathrm{ks}$, plus other small scale variability afterwards. This demonstrates that even during the quiescent state some degree of X-ray variability was present. When modeling the plasma with two absorbed thermal APEC components, the cool component was around 0.8$0.9 \mathrm{keV}$ and the hot component in $1.9 \mathrm{keV}<k T<2.7 \mathrm{keV}$, which is within $2 \sigma$ of the values of hot temperatures seen 


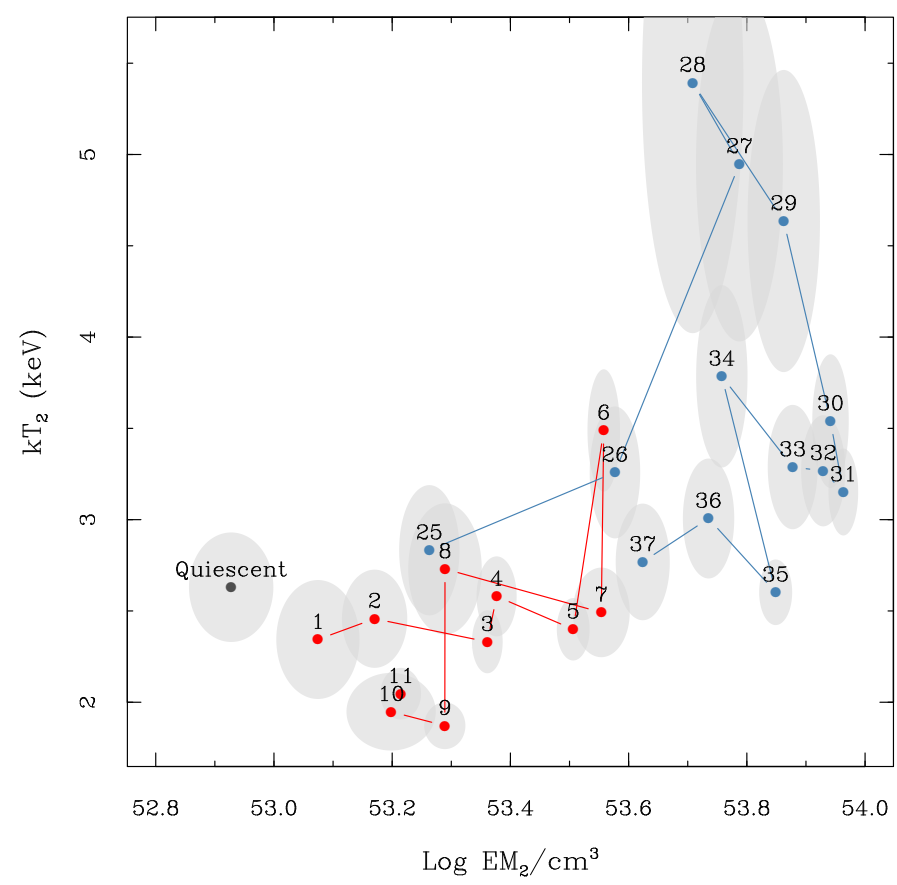

Fig. 6. $k T_{2}$ vs. emission measure $\left(\mathrm{EM}_{2}\right)$ of the hot component of the $2 \mathrm{~T}$ APEC best-fit models for the time intervals (see text) relative to the first event (red points) and second event (blue points). The point relative to the quiescent phase (time intervals 12-24) corresponds to the hottest component of the $3 \mathrm{~T}$ VAPEC best-fit model. Ellipses denote the $1 \sigma$ confidence ranges.

during the first event. On average, the quiescent flux is about $1.35 \times 10^{-12} \mathrm{erg} \mathrm{s}^{-1} \mathrm{~cm}^{-2}$, which is very similar to the quiescent flux measured in $2013\left(1.5 \times 10^{-12} \mathrm{erg} \mathrm{s}^{-1} \mathrm{~cm}^{-2}\right)$.

We grouped together the time bins between 11 and 24 (interval 13 excluded) to gain more count statistics and allow a more refined analysis of the corresponding spectrum (black curve in Fig. 5). We tried models with two, three, and four APEC components and models with two and three VAPEC components. The 2T APEC model does not give us a statistically valid fit, while the three APEC component model does. The model with four APEC components does not improve the fit to data. For the three APEC components we find $k T_{1}=0.26 \pm 0.03 \mathrm{keV}$, $k T_{2}=0.98 \pm 0.04 \mathrm{keV}$, and $k T_{3}=2.97 \pm 0.7 \mathrm{keV}$ with ratios of emission measures $(\mathrm{EM}) E M_{3} / E M_{1} \sim 0.12, E M_{2} / E M_{1} \sim 0.4$. We thus detect a soft component at $0.26 \mathrm{keV}$ and there is evidence of a hot component at $\sim 3 \mathrm{keV}$ also during quiescence.

For the VAPEC models we left $\mathrm{Fe}, \mathrm{O}$, and $\mathrm{Ne}$ free to vary and linked all other elements to the $\mathrm{Fe}$ abundance. Both the 2T VAPEC model and the 3T VAPEC give a satisfactory fit to the data. For the 2T VAPEC model the two temperatures are $k T_{1}=(0.81 \pm 0.04) \mathrm{keV}$ and $k T_{2}=(2.6 \pm 0.5) \mathrm{keV}$, respectively, with $E M_{2} / E M_{1} \sim 0.5$. The abundances of Fe and other metals are found around $0.13 Z_{\odot}$, but with $\mathrm{O}$ and $\mathrm{Ne}$ around 1.1 and 0.75 times the solar value. This pattern is similar to that observed in active stellar coronae (the so-called inverse FIP effect, see, e.g., Güdel 2004 and references therein) where the abundance of $\mathrm{Fe}$ (an element with low first ionization potential, FIP) is found depleted with respect to the value measured in the solar corona, while the abundances of elements with high FIP, such as $\mathrm{O}$ and $\mathrm{Ne}$, appear enhanced.

For the 3T VAPEC model, a pattern of abundances similar to that of the 2T VAPEC model fit is found $\left(\mathrm{Fe} / \mathrm{Fe}_{\odot}=0.15\right.$, $[\mathrm{O} / \mathrm{Fe}] \sim 1.3,[\mathrm{Ne} / \mathrm{Fe}] \sim 0.8)$. In this case the temperatures are $k T_{1}=(0.1 \pm 0.1) \mathrm{keV}, k T_{2}=(0.80 \pm 0.04) \mathrm{keV}$, and $k T_{3}=(2.63 \pm 0.6) \mathrm{keV}$ with $\mathrm{EM}$ ratios of $E M_{2} / E M_{1} \sim 4.5$, $E M_{3} / E M_{1} \sim 1.9$. We detect thus a cool component and a hot component in the quiescent phase spectrum, whereas the main component remains that at $\sim 0.8 \mathrm{keV}$. This also justifies the use of a simple 2T APEC model for time resolved spectroscopy, and the focus on the hot component of such a model to study the two main increases of X-ray flux of $\rho$ Oph A.

Second event (flare, intervals 25-37). These intervals contain the second and most powerful event observed in $\rho$ Oph A. The $p n$ spectrum relative to the time bins around the peak of the rate is shown in Fig. 5 (green curve). The spectrum is characterized by the appearance of lines of highly ionized Fe at $6.7 \mathrm{keV}$. The rise of the flare started at interval number 25 , and its evolution is best described by the hot component of the spectral fitting, which we discuss here. An increase of EM and temperature is detected, starting from intervals 25 and 26, and even more during intervals 27-29. Interval number 28 shows the peak of $k T \sim 5.4 \mathrm{keV}$, then $k T$ decreases steadily through intervals 29-31, and more slowly through intervals 32-37, with some apparent reheating at interval 34. At these times EM showed its maximum values (with a peak of $\log \mathrm{EM}\left(\mathrm{cm}^{-} 3\right) \geq 53.9$ in interval 31$)$ then it decreased toward pre-flare values. The peak of the hot temperature was reached in interval 28 and does not coincide with the maximum of EM, which peaked during interval 31. This fact is consistent with what is observed in the flares of cool stars and in the Sun, where the initial heating and peak of temperature is followed by an increase of the density of the flaring loop, filled up by plasma evaporating from the loop footpoints (Reale 2007).

The behavior of the flare closely resembles that of solar and stellar flares observed in X-rays, where energy is suddenly released with an impulse in plasma magnetically confined in loops, followed by a longer cooling decay. We presume that $X M M$ Newton did not completely observe the full decay of the flare and the disappearance of the hot region as in the first event. This and the too shallow track of the decay in the EM $-k T$ diagram did not allow us to have reliable diagnostics from the flare decay as in Reale et al. (1997), and we preferred to derive loop diagnostics from the analysis of the rise phase, as described in Reale (2007). The loop length can be derived from measuring the duration of the rise of the light curve and/or the time delay of the emission measure peak from the temperature peak (Reale 2007) as follows:

$$
\begin{aligned}
& L_{9} \approx 3 \psi^{2} T_{0,7}^{1 / 2} t_{M, 3} \approx 190 \\
& L_{9} \approx 2.5 \frac{\psi^{2}}{\ln \psi} T_{0,7}^{1 / 2} \Delta t_{0-M, 3} \approx 140,
\end{aligned}
$$

where

$\psi=\frac{T_{0}}{T_{M}} \approx 1.9$,

$L_{9}$ is the loop half-length in units of $10^{9} \mathrm{~cm}, t_{M, 3} \approx 4.6 \mathrm{ks}$ is the time of the emission measure maximum measured from the flare beginning, $T_{0}\left(T_{0,7}\right)$ the loop maximum temperature (in units of $10^{7} \mathrm{~K}$ ), $T_{M}$ the temperature at the emission measure maximum, and $\Delta t_{0-M, 3} \approx 2.6$ the time elapsed between the peak of the temperature and the peak of the emission measure in ks. The values $T_{0} \approx 150 \mathrm{MK}$ (bin 28) and $T_{M} \approx 80 \mathrm{MK}$ (bin 31) are maximum loop temperatures inferred from the measured temperatures, which average over the whole flaring loop (see Appendix in Reale 2007). In summary, we inferred a loop half-length $L=1.4-1.9 \times 10^{11} \mathrm{~cm}$, which corresponds to about 

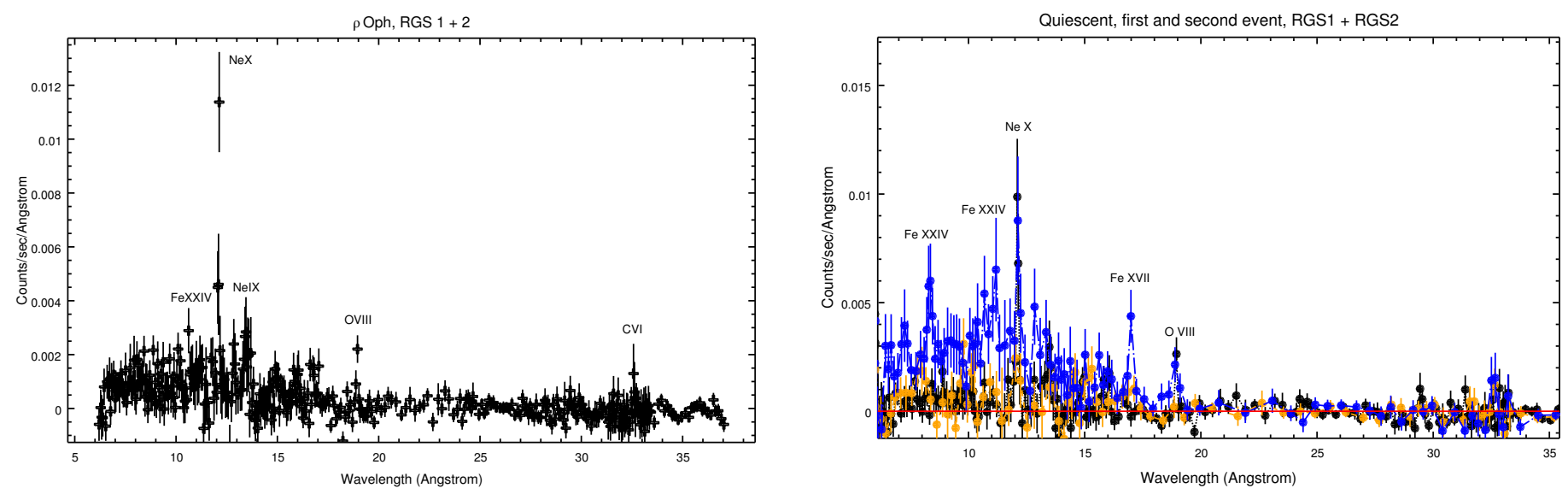

Fig. 7. RGS spectra of $\rho$ Oph A in the range 5-30 A. Left panel: average spectrum during the full exposure (140 ks). The spectrum was binned to have at least 25 counts per bin. Right panel: black refers the spectrum relative to the quiescent state between the two main variability events, the orange spectrum refers to the first event, and the blue spectrum refers to the second event. Changes in the intensities of lines are visible mostly during the flare event at wavelengths below $12 \AA$, when both lines and continuum increased their strength.

$25 \%-33 \%$ of the stellar radius or about $2-2.7$ times the solar radius. We also estimated that the coronal magnetic field must have an intensity $B \geq 300 \mathrm{G}$ to confine the plasma within the loop.

\section{RGS spectra}

The average spectrum of $\rho$ Oph A is shown in Fig. 7 (left panel), while the spectra of the quiescent (40-115 ks, black) interval, the first (10-40 ks, orange) and second (115-140 ks, blue) event are shown in the right panel of Fig. 7. These spectra are rebinned to have at least 25 counts per bin to enhance the signal, although this reduces the original spectral resolution. Qualitatively, hot lines from $\mathrm{O}$ VIII and Ne X are detected both in the quiescent interval and during the variability events, indicating the presence of hot plasma $(k T \geq 1 \mathrm{keV})$ that is already detected in the $p n$ spectra. Another sign of high temperature plasma is the relatively high level of continuum, below $10 \AA$, similar to what has been observed in stars with high activity and plasma temperatures like, for example, UX Ari and $\epsilon$ Eri (Ness et al. 2002).

The best fit of the average RGS spectrum with two absorbed APEC thermal components results in a temperature around $0.3 \mathrm{keV}$ and a hot temperature around $5 \mathrm{keV}$ for a fixed $Z=$ $0.3 Z_{\odot}$, in agreement with the $p n$ spectral analysis, even though the range of energy of the RGS is limited to $\sim 2.5 \mathrm{keV}$.

Changes of line intensities are mostly observed during the second event. In particular, we observed an increase of both continuum and line strength at wavelengths shorter than $12 \AA$ during the second (flare) event. Lines of Fe XXIV are visible during the flare owing to temperatures in excess of $5 \mathrm{keV}$ as determined from the analysis of the $p n$ spectra.

We measured the line fluxes by modeling single lines with Gaussian profiles characterized by a line central wavelength and a full width at half maximum (FWHM). In this way we take into account any intrinsic widths of the lines. The model also comprises a continuum level in a window of about $0.7-1 \AA$ around each feature; we used CIAO Sherpa $4.8^{2}$ Table A.2 presents the measurements of the main lines identified in the spectra. Determining a $1 \sigma$ confidence range was difficult for some lines as it depends on the statistics and number of bins available for fitting. As a consequence, any quantitative conclusions on the widths

\footnotetext{
2 http://cxc.harvard.edu/sherpa4.8/ to obtain the best fit pa-
} rameters. of the lines and the amount of change during the increase of rates are impossible with these data. In general the line widths are consistent with zero even for the spectra relative to the high rate events, when we presume that a line broadening should be present owing to the traveling of the source of X-rays across the surface, either this is an active spot or an unseen companion during the interval in which the spectra were accumulated. Qualitatively, RGS spectra show the potential for a deeper high resolution spectroscopic follow up of $\rho$ Oph in order to improve the statistics and precisely measure line widths and velocity fields.

\section{Discussion and conclusions}

We have presented an XMM-Newton observation that monitored $\rho$ Oph for $140 \mathrm{ks}$, encompassing an entire rotation period of the primary star of the system $(P \sim 1.2$ days). We observed a change of X-ray emission twice, each time lasting about $35 \mathrm{ks}$ and separated by about $104 \mathrm{ks}$. This "lighthouse" effect has increased its strength in the 2016 observation. On the other hand, the quiescent phase has remained at similar levels of emission with respect to the 2013 observation. The cause of this phenomenon can be attributed either to a large active spot on the stellar surface, or an unknown low mass companion, and both scenarios have strengths and weaknesses.

Active spot. In this scenario, the spot is a magnetospheric feature that could also have a photospheric counterpart and has been steadily present on the stellar surface for at least $2.5 \mathrm{yr}$ (i.e., since the epoch of the 2013 XMM-Newton observation, Paper I), as the light curves of 2013 and 2016 can be put in phase with the stellar rotation period, thus matching the periodic increases of rate. In this respect $\rho$ Oph A would be a magnetic early B star similar to those discovered by, for example, Fossati et al. (2014). From the duration of the rate change (about 30-35 ks) compared to the period (104 ks) and taking into account the inclination angle of the star $\left(i \sim 45^{\circ}\right)$, we infer a quite large size for the spot, amounting to about 1.5 stellar radii. The cusp-like shape of the first episode of variability, free of major flares, and the lack of a flat top level suggest that such a large spot could be plausible because when the spot is completely on view after emerging from one limb it starts to disappear at the opposite limb.

We attribute the origin of the spot to a strong magnetic field that is responsible for creating the spot in the form of magnetic loops. The loops confine high temperature plasma that emits 
X-rays. In this scenario the spot can be seen as a scaled-up version of loop-populated solar active regions. Flares can occur within these loops that make the typical temperature of the plasma rise above $k T \geq 5 \mathrm{keV}$, as observed in the second event. No constraints on the magnetic field in $\rho$ Oph A could be found in the literature, therefore magnetic field scenario remains hypothetical at the moment. In the future work we aim to obtain reliable magnetic field measurements of this interesting object.

If $\rho$ Oph A has a magnetic field, then it would not be the only known early B star exhibiting surface spots and a magnetic field. In $\tau$ Sco, Donati et al. (2006) discovered a magnetic field with a complex topology. The magnetic loops should be responsible for emitting X-rays as in solar-type stars. However, the lack of modulated X-ray emission hints that the magnetic loops are small and distributed across the surface (Oskinova 2016; Ignace et al. 2010). However, in $\rho$ Oph we find well-defined periodic modulation, thus a strong magnetic field must be concentrated in a large spot. We speculate that the magnetic field has a large scale structure that is similar to a simple dipolar configuration and a stable configuration deduced by the long lifetime of the spot $(\geq 2.5 \mathrm{yr})$.

Wade et al. (2017) have found a strong magnetic field, on the order of a few $\mathrm{kG}$, in HD $164492 \mathrm{C} 1$, which is an early B star similar to $\rho$ Oph rotating in 1.36 days and part of a hierarchical system with other two A/Herbig Ae stars belonging to the Trifid nebula. However, one difference from $\rho$ Oph is that the HD 164492 system is tighter than that of $\rho$ Oph and the origin of the magnetic field in such objects could be associated with binarity and the stellar formation mechanism. Another case of strong magnetism in an early B star (CPD -62 2124$)$ is reported by Hubrig et al. (2017), where a strong dipolar magnetic field with strength in excess of $\sim 30 \mathrm{kG}$ has been detected. Analogous to $\rho \mathrm{Oph}$ A, CPD $-62^{\circ} 2124$ is a fast rotator with rotational period of about 1.45 days.

Weak emission of X-rays has been detected in $\sigma$ Sgr (B2V, $\sim 65 \mathrm{pc}$ ) in a short $10 \mathrm{ks}$ XMM-Newton observation (PI Oskinova; XMM ObsId 0721210101). For this star we derived a flux of about $7.4 \times 10^{-15} \mathrm{erg} \mathrm{s}^{-1} \mathrm{~cm}^{-2}$ in $0.3-8.0 \mathrm{keV}$ and a luminosity of $\sim 3.7 \times 10^{27} \mathrm{erg} \mathrm{s}^{-1}$, which is about three orders of magnitude lower than the X-ray luminosity of $\rho$ Oph A. The count statistics are low but we can infer that the spectrum is almost entirely below $1 \mathrm{keV}$ and peaks around $0.7 \mathrm{keV}$, pointing to a substantial difference in the mechanisms that produce X-rays in $\sigma \mathrm{Sgr}$ and $\rho$ Oph.

B stars do not possess magnetic coronae such as solar-type stars, and in this respect they are similar to A type stars, which are mostly dark in X-rays and lack strong magnetic fields. However, even among A type stars, which are presumed to be less active stars during the main sequence lifetime because they lack a solar-type corona, Balona (2013) found a number of stars with spots in an analysis based on Kepler data.

Low mass companion. Another explanation is the presence of an unknown low mass stellar companion as the source of X-rays. If such a companion is fully responsible for the X-rays, it means that it is not completely eclipsed by $\rho$ Oph A when orbiting around it. To support this we notice that $\rho$ Oph B (another B2 star) is undetected in the same data. During the quiescent state we observed the less active part of the corona of the unknown companion, while during the recurrent increase of rate we had observed the most active part of it, i.e., perhaps the part of the surface of the companion facing the primary (its day side). From the combined analysis of the X-ray derived period and the $v \sin i$ from the line profile, it is suggested that the primary is inclined by $\sim 45^{\circ}$. If the plane of the orbit of the companion has the same inclination it is plausible that during the superior conjunction part of its surface (its day side) is partially obscured by the primary, dark in X-rays.

Two factors can boost the X-ray activity of the hypothetical companion to such a high level: its young age, coeval with $\rho$ Oph and with the other solar-type stars discovered around it (Pillitteri et al. 2016), and being part of a tight binary system. This would enhance the stellar activity even more than in a configuration of single star, as observed for example in the RS CVn systems. In fact, if the companion rotates in 1.2 days, as $\rho$ Ophs does, such a period would correspond to a very close separation of $\sim 9.8 R_{\odot}$ that is similar to the radius of $\rho \mathrm{Oph} \mathrm{A}$. This results in an extreme system with the companion almost on the verge of collapsing onto the primary. The system should be in a 1:1 spinorbit locked configuration reached in the first $5 \mathrm{Myr}$, making its evolution fast.

The plasma temperatures, the type of impulsive variability and the pattern of abundances of the quiescent spectrum are fully consistent with the X-ray properties of young, active pre-main sequence stars. If a young unseen companion emits such X-rays, the derived loop length $\left(1.4-1.9 \times 10^{11} \mathrm{~cm}\right.$ or $\left.\sim 2-2.7 R_{\odot}\right)$ would be a few of its stellar radius. This implies an extended structure that perhaps could connect the surface of $\rho$ Oph A with the companion and hints to some form of magnetic interaction between the two stars. At the same time the hottest part of the corona of the companion should be eclipsed at some phases of the orbital motion to reproduce the features observed in the light curve.

In the literature we find cases of X-rays from massive stars that are attributed to a low mass companion, such as $\sigma$ Ori E (B2Vp; Sanz-Forcada et al. 2004), which was detected in a flaring state during a XMM-Newton observation. $\sigma$ Ori $\mathrm{E}$ has a hard spectrum similar to $\rho \mathrm{Oph} \mathrm{A}$ and remarkably harder than that of $\sigma$ Ori $\mathrm{AB}(\mathrm{O} 9.5 \mathrm{~V})$. However, in contrast to $\sigma$ Ori $\mathrm{E}$ we did not detect a $6.4 \mathrm{keV}$ line from neutral $\mathrm{Fe}$, even during interval 28 when the spectrum reached the maximum temperature. The absence of a circumstellar disk on the companion of $\rho$ Oph A is perhaps linked to the lack of the $6.4 \mathrm{keV}$ line as seen in $\sigma$ Ori E. There, the neutral Fe could emit by fluorescence during the flare as the relatively cold material of the disk is hit by high energy $\mathrm{X}$-ray photons created during the flare, while in $\rho$ Oph A there is no circumstellar disk from where fluorescence of neutral Fe can arise.

The analysis of the optical spectra from the ESO archive did not reveal any Doppler shift due to the presence of the companion. This does not exclude its presence but rather calls for an ad hoc spectroscopic campaign on $\rho$ Oph A.

A mixture of both scenarios could be present as well; that is, $\mathrm{X}$-rays produced by the coronal activity of an unseen companion coupled to and enhanced by an intrinsic magnetism of $\rho$ Oph A.

Among other cases of uncertain origin of X-rays we cite IQ Aur, which is an A0p bright in X-rays and flaring during a XMM-Newton observation with temperatures up to $6 \mathrm{keV}$ (Robrade \& Schmitt 2011). In the case of IQ Aur a magnetized wind model and the presence of an unseen low mass companion are discussed by Robrade et al. Qualitatively the X-rays from IQ Aur are similar to those from $\rho$ Oph except for the fact that the flare has been observed once and never with periodic recurrence. In the case of a magnetized wind emission the occurrence of a flare does not easily fit the current models.

Other mechanisms of production of X-rays observed in binary systems of massive stars are less probable because the separation between $\rho$ Oph A and B is about $300 \mathrm{AU}$, the short period of the phenomenon appears to be linked to the rotation of the primary, and because the winds from B2 stars are weaker than those from $\mathrm{O}$ and Wolf Rayet stars. 
A clear case of periodic variation of X-ray flux of a massive star has been reported in $\chi^{1}$ CMa by Oskinova et al. (2014). $\chi^{1}$ CMa is a variable Beta Cep type star (B0.5-1 V-IV) characterized by a strong magnetic field $(B>5000 \mathrm{G}) \cdot \chi^{1}$ CMa exhibits X-ray and $\mathrm{H}$-band mag variability in phase, however its X-ray spectrum is softer than $\rho$ Oph and it does not show flares. The mechanism that produces X-rays in $\chi^{1} \mathrm{CMa}$ is still not completely clear; it is likely linked to the compression phase and the plasma heating in the consequent shock, as the maximum rate of X-rays happens at the minimum stellar radius. This scenario is not applicable to $\rho$ Oph because it cannot explain the much higher plasma temperatures observed in it and its flaring activity.

The present work indicates the peculiarity of $\rho$ Oph in the $\mathrm{X}$-ray band, as it represents perhaps the best example of an X-ray active early B star and one of the most favorable targets for studying magnetism in B stars. On the other hand, $\rho$ Oph A could be an extreme system with a low mass companion on the verge of collapsing onto the primary. In both cases $\rho$ Oph A deserves more investigation to understand the origin of its X-rays.

Acknowledgements. We would like to thank the anonymous referee for constructive comments. I.P. is grateful to dr. Javier Lopez-Santiago and dr. Ines CrespoChacon for providing useful information on the analysis of the line profile and the derivation of $v \sin i$. S.J.W. was supported by NASA contract NAS8-03060.

\section{References}

Babel, J., \& Montmerle, T. 1997, ApJ, 485, L29

Balona, L. A. 2013, in Progress in Physics of the Sun and Stars: A New Era in Helio- and Asteroseismology, eds. H. Shibahashi, \& A. E. Lynas-Gray, ASP Conf. Ser., 479, 385
Donati, J.-F., Howarth, I. D., Jardine, M. M., et al. 2006, MNRAS, 370, 629 Feldmeier, A., Kudritzki, R.-P., Palsa, R., Pauldrach, A. W. A., \& Puls, J. 1997a, A\&A, 320, 899

Feldmeier, A., Puls, J., \& Pauldrach, A. W. A. 1997b, A\&A, 322, 878

Fossati, L., Zwintz, K., Castro, N., et al. 2014, A\&A, 562, A143

Fossati, L., Castro, N., Schöller, M., et al. 2015, A\&A, 582, A45

Glebocki, R., \& Gnacinski, P. 2005, VizieR Online Data Catalog: III/244

Gray, D. F. 1992, The Observation and Analysis of Stellar Photospheres (Cambridge University Press)

Güdel, M. 2004, A\&ARv, 12, 71

Hubrig, S., Kholtygin, A. F., Ilyin, I., Schöller, M., \& Jarvinen, S. P. 2017, MNRAS, submitted [arXiv: 1702.01063]

Ignace, R., Oskinova, L. M., Jardine, M., et al. 2010, ApJ, 721, 1412

Malkov, O. Y., Tamazian, V. S., Docobo, J. A., \& Chulkov, D. A. 2012, A\&A, 546, A69

Ness, J.-U., Schmitt, J. H. M. M., Burwitz, V., et al. 2002, A\&A, 394, 911

Nieva, M.-F., \& Przybilla, N. 2012, A\&A, 539, A143

Oskinova, L. M. 2016, Adv. Space Res., 58, 739

Oskinova, L. M., Nazé, Y., Todt, H., et al. 2014, Nat. Comm., 5, 4024

Owocki, S. P., Cranmer, S. R., \& Gayley, K. G. 1998, Ap\&SS, 260, 149

Pillitteri, I., Wolk, S. J., Goodman, A., \& Sciortino, S. 2014, A\&A, 567, L4

Pillitteri, I., Wolk, S. J., Chen, H. H., \& Goodman, A. 2016, A\&A, 592, A88 Reale, F. 2007, A\&A, 471, 271

Reale, F., Betta, R., Peres, G., Serio, S., \& McTiernan, J. 1997, A\&A, 325, 782

Robrade, J., \& Schmitt, J. H. M. M. 2011, A\&A, 531, A58

Sanz-Forcada, J., Franciosini, E., \& Pallavicini, R. 2004, A\&A, 421, 715

Smith, M. A., \& Gray, D. F. 1976, PASP, 88, 809

Tonry, J., \& Davis, M. 1979, AJ, 84, 1511

ud-Doula, A., \& Owocki, S. P. 2002, ApJ, 576, 413

Uesugi, A., \& Fukuda, I. 1982, VizieR Online Data Catalog: III/63B

van Belle, G. T. 2012, A\&ARv, 20, 51

van Leeuwen, F. 2007, A\&A, 474, 653

Wade, G. A., Petit, V., Grunhut, J., \& Neiner, C. 2014, ArXiv e-prints [arXiv: 1411.6165]

Wade, G. A., Shultz, M., Sikora, J., et al. 2017, MNRAS, 465, 2517 
I. Pillitteri et al.: The early B-type star Rho Ophiuchi A is an X-ray lighthouse

\section{Appendix A: Time resolved spectroscopy}

Table A.1. Best-fit parameters from time resolved spectroscopy of the first event and second event.

\begin{tabular}{|c|c|c|c|c|c|c|c|c|c|c|c|c|c|c|c|c|}
\hline $\begin{array}{r}\text { Interval } \\
\text { First event }\end{array}$ & $\begin{array}{r}\text { Start } \\
\text { ks }\end{array}$ & $\begin{array}{r}\text { End } \\
\text { ks }\end{array}$ & $\mathrm{T} 1$ & \multicolumn{2}{|c|}{$\mathrm{keV}$} & Err (T2) & \multicolumn{2}{|c|}{$\log \mathrm{cm}^{-3}$} & $\begin{array}{c}F_{\mathrm{s}} \\
10^{-1}\end{array}$ & $\begin{array}{r}F_{\mathrm{h}} \\
\mathrm{erg} \mathrm{s}^{-1}\end{array}$ & $\begin{array}{r}F_{\text {tot }} \\
\mathrm{cm}^{-2}\end{array}$ & $\begin{array}{r}\log L_{X} \\
\mathrm{erg} \mathrm{s}^{-1}\end{array}$ & HR & $x^{2}$ & d.o.f. & $P\left(\chi^{2}>\chi_{0}^{2}\right)$ \\
\hline 1 & 0.00 & 5.45 & 0.89 & 0.06 & 2.34 & 0.33 & $52.94_{-0.07}^{0.06}$ & $53.00_{-0.08}^{0.06}$ & 7.66 & 4.77 & 15.63 & 30.36 & 0.62 & 1.22 & 36 & 16.75 \\
\hline 2 & 5.45 & 10.76 & 0.87 & 0.06 & 2.46 & 0.27 & $52.90_{-0.07}^{0.06}$ & $53.09_{-0.06}^{0.05}$ & 8.00 & 5.80 & 17.19 & 30.40 & 0.73 & 1.25 & 38 & 13.96 \\
\hline 3 & 10.76 & 15.20 & 0.61 & 0.05 & 2.33 & 0.17 & $52.90_{-0.05}^{0.04}$ & $53.29_{-0.03}^{0.03}$ & 10.17 & 7.73 & 21.92 & 30.51 & 0.76 & 1.12 & 38 & 28.36 \\
\hline 4 & 15.20 & 19.04 & 0.83 & 0.05 & 2.58 & 0.22 & $52.95_{-0.05}^{0.04}$ & $53.30_{-0.04}^{0.03}$ & 10.67 & 9.22 & 24.49 & 30.56 & 0.86 & 1.35 & 38 & 7.19 \\
\hline 5 & 19.04 & 22.44 & 0.73 & 0.07 & 2.40 & 0.17 & $52.85_{-0.07}^{0.06}$ & $53.43_{-0.03}^{0.03}$ & 11.64 & 11.05 & 27.93 & 30.61 & 0.95 & 1.61 & 38 & 0.98 \\
\hline 6 & 22.44 & 25.05 & 0.81 & 0.06 & 3.49 & 0.33 & $53.03_{-0.05}^{0.05}$ & $53.48_{-0.03}^{0.03}$ & 14.27 & 17.20 & 37.65 & 30.74 & 1.21 & 1.17 & 40 & 21.82 \\
\hline 7 & 25.05 & 27.74 & 0.89 & 0.08 & 2.49 & 0.25 & $53.06_{-0.1}^{0.08}$ & $53.48_{-0.05}^{0.05}$ & 14.61 & 13.43 & 34.81 & 30.71 & 0.92 & 1.13 & 39 & 26.03 \\
\hline 8 & 27.74 & 31.24 & 0.95 & 0.04 & 2.73 & 0.36 & $53.16_{-0.06}^{0.05}$ & $53.21_{-0.07}^{0.06}$ & 11.79 & 8.99 & 26.14 & 30.59 & 0.76 & 1.89 & 36 & 0.10 \\
\hline 9 & $3: 1.24$ & 36.05 & 0.79 & 0.05 & 1.87 & 0.13 & $52.90_{-0.05}^{0.05}$ & $53.21_{-0.04}^{0.03}$ & 9.36 & 5.70 & 19.20 & 30.45 & 0.61 & 0.88 & 36 & 66.73 \\
\hline 10 & 36.05 & 41.22 & 0.88 & 0.09 & 1.95 & 0.21 & $52.91_{-0.1}^{0.09}$ & $53.12_{-0.08}^{0.07}$ & 8.33 & 5.09 & 17.18 & 30.40 & 0.61 & 1.56 & 35 & 1.91 \\
\hline 11 & 41.22 & 46.68 & 0.74 & 0.05 & 2.05 & 0.14 & $52.86_{-0.05}^{0.04}$ & $53.14_{-0.04}^{0.03}$ & 8.34 & 5.18 & 16.91 & 30.40 & 0.62 & 1.48 & 35 & 3.28 \\
\hline \multicolumn{17}{|l|}{ Second event } \\
\hline 25 & 115.76 & 120.09 & 0.90 & 0.06 & 2.83 & 0.36 & $52.98_{-0.07}^{0.06}$ & $53.19_{-0.05}^{0.05}$ & 9.47 & 8.05 & 21.65 & 30.50 & 0.85 & 1.14 & 38 & 26.03 \\
\hline 26 & 120.09 & 122.48 & 1.01 & 0.05 & 3.26 & 0.36 & $53.13_{-0.08}^{0.07}$ & $53.50_{-0.04}^{0.04}$ & 14.54 & 17.91 & 39.91 & 30.77 & 1.23 & 1.15 & 40 & 24.14 \\
\hline 27 & 122.48 & 123.99 & 1.26 & 0.13 & 4.95 & 0.97 & $53.43_{-0.2}^{0.1}$ & $53.71_{-0.08}^{0.07}$ & 20.61 & 37.10 & 69.06 & 31.01 & 1.80 & 0.81 & 43 & 81.24 \\
\hline 28 & 123.99 & 125.29 & 1.22 & 0.08 & 5.39 & 1.37 & $53.59_{-0.1}^{0.09}$ & $53.63_{-0.09}^{0.08}$ & 24.51 & 36.62 & 75.48 & 31.05 & 1.49 & 1.21 & 39 & 17.76 \\
\hline 29 & 125.29 & 126.57 & 1.11 & 0.18 & 4.64 & 0.83 & $53.35_{-0.2}^{0.1}$ & $53.79_{-0.07}^{0.06}$ & 24.07 & 42.29 & 79.74 & 31.07 & 1.76 & 0.96 & 43 & 54.67 \\
\hline 30 & 126.57 & 127.81 & 0.91 & 0.09 & 3.54 & 0.37 & $53.20_{-0.1}^{0.09}$ & $53.87_{-0.03}^{0.03}$ & 27.33 & 41.33 & 82.12 & 31.08 & 1.51 & 1.05 & 41 & 37.73 \\
\hline 31 & 127.81 & 129.03 & 0.78 & 0.08 & 3.15 & 0.24 & $53.20_{-0.08}^{0.06}$ & $53.89_{-0.03}^{0.02}$ & 29.77 & 39.43 & 83.03 & 31.09 & 1.32 & 0.83 & 40 & 76.04 \\
\hline 32 & 129.03 & 130.35 & 1.02 & 0.06 & 3.27 & 0.30 & $53.34_{-0.1}^{0.08}$ & $53.85_{-0.04}^{0.04}$ & 28.62 & 39.28 & 82.93 & 31.09 & 1.37 & 0.91 & 42 & 62.93 \\
\hline 33 & 130.35 & 131.57 & 1.03 & 0.05 & 3.29 & 0.34 & $53.46_{-0.08}^{0.06}$ & $53.80_{-0.04}^{0.04}$ & 29.63 & 36.34 & 81.30 & 31.08 & 1.23 & 0.84 & 39 & 75.48 \\
\hline 34 & 131.57 & 132.93 & 0.93 & 0.05 & 3.78 & 0.50 & $53.49_{-0.06}^{0.05}$ & $53.68_{-0.05}^{0.04}$ & 29.04 & 30.65 & 72.59 & 31.03 & 1.06 & 0.82 & 40 & 78.41 \\
\hline 35 & 132.93 & 134.49 & 0.79 & 0.07 & 2.60 & 0.18 & $53.18_{-0.07}^{0.06}$ & $53.77_{-0.03}^{0.03}$ & 25.03 & 26.31 & 62.84 & 30.97 & 1.05 & 1.00 & 41 & 47.05 \\
\hline 36 & 134.49 & 136.14 & 1.04 & 0.05 & 3.01 & 0.33 & $53.33_{-0.08}^{0.06}$ & $53.66_{-0.05}^{0.04}$ & 21.66 & 24.75 & 57.82 & 30.93 & 1.14 & 1.10 & 39 & 30.78 \\
\hline 37 & 136.14 & 138.19 & 0.98 & 0.05 & 2.77 & 0.32 & $53.29_{-0.07}^{0.06}$ & $53.55_{-0.05}^{0.04}$ & 18.98 & 18.28 & 46.58 & 30.84 & 0.96 & 1.19 & 38 & 20.05 \\
\hline
\end{tabular}

Notes. We list the temporal intervals, temperatures, logarithm of the emission measures (EM), unabsorbed fluxes in bands $0.3-1.0 \mathrm{keV}\left(F_{\mathrm{s}}\right)$, $1.5-8.0 \mathrm{keV}\left(F_{\mathrm{h}}\right), 0.3-8.0 \mathrm{keV}\left(F_{\text {tot }}\right)$, unabsorbed X-ray luminosity in $0.3-8.0 \mathrm{keV}$, hardness ratio $\left(\mathrm{HR}=F_{\mathrm{h}} / F_{\mathrm{s}}\right)$, and $\chi^{2}$ statistics. 
A\&A 602, A92 (2017)

Table A.2. Lines measured in the RGS spectra relative to the full exposure, quiescent, and high state.

\begin{tabular}{|c|c|c|c|c|}
\hline Ion & & Full & Quiescent & High state \\
\hline Fe XXIV & $F W H M(\AA)$ & $0.004_{-0.004}^{0.288}$ & - & $0.08_{-0.08}^{0.17}$ \\
\hline \multirow[t]{2}{*}{$8.0 \AA$} & pos. $(\AA)$ & $8.08_{-0.04}^{0.40}$ & - & $8.35_{-0.12}^{0.04}$ \\
\hline & int. (ct/ks) & $0.6_{-0.6}^{0.6}$ & - & $0.11_{-0.08}^{3.67}$ \\
\hline Fe XXIV & $F W H M(\AA)$ & $0.011_{-}^{0.061}$ & $0.01-^{0.22}$ & - \\
\hline \multirow[t]{2}{*}{$10.6 \AA$} & pos. $(\AA)$ & $10.64_{-0.017}^{0.016}$ & $10.64-$ & - \\
\hline & int. (ct/ks) & $0.33_{-0.3}^{1.4}$ & $0.04_{-0.15}^{0.15}$ & - \\
\hline $\operatorname{Ne} \mathrm{X}$ & $F W H M(\AA)$ & $0.0016_{-0.0004}^{0.0442}$ & $0.012_{-}^{0.05}$ & $0.005_{-0.003}^{0.005}$ \\
\hline \multirow[t]{2}{*}{$12.1 \AA$} & pos. $(\AA)$ & $12.12_{-0.01}^{0.01}$ & $12.11_{-0.01}^{0.01}$ & $12.13_{-0.01}^{0.01}$ \\
\hline & int.(ct/ks) & $6.0_{-5.0}^{8.0}$ & $0.82_{-0.07}^{1.63}$ & $1.5_{-1.3}^{-}$ \\
\hline Ne IX & $F W H M(\AA)$ & $0.10_{-0.1}^{0.04}$ & $0.29_{-0.28}^{0.24}$ & $0.004_{-}^{0.14}$ \\
\hline \multirow[t]{2}{*}{$13.4 \AA$} & pos. $(\AA)$ & $13.44_{-0.05}^{0.05}$ & $13.43_{-0.09}^{0.09}$ & $13.42_{-}^{-}$ \\
\hline & int.(ct/ks) & $0.036_{-}^{3.0}$ & $0.026_{-0.014}^{10.000}$ & $0.39_{-1.4}^{3.5}$ \\
\hline Fe XVII & $F W H M(\AA)$ & $0.10_{-0.10}^{0.04}$ & $0.15_{-0.15}^{0.10}$ & $0.001_{-}^{0.15}$ \\
\hline \multirow[t]{2}{*}{$15 \AA$} & pos. $(\AA)$ & $14.97_{-0.07}^{0.05}$ & $14.92_{-0.07}^{0.06}$ & $15.03_{-0.03}^{0.04}$ \\
\hline & int. (ct/ks) & $0.04_{-0.03}^{4.6}$ & $0.03_{-0.02}^{27.00}$ & $4.0_{-1.1}^{3.0}$ \\
\hline O VIII & $F W H M(\AA)$ & $0.07_{-0.07}^{0.03}$ & $0.06_{-0.06}^{0.02}$ & $0.14_{-0.14}^{0.07}$ \\
\hline \multirow[t]{2}{*}{$18.9 \AA$} & pos. $(\AA)$ & $18.95_{-0.02}^{0.02}$ & $18.95_{-0.03}^{0.03}$ & $18.92_{-0.04}^{0.04}$ \\
\hline & int. (ct/ks) & $0.084_{-0.031}^{2.71}$ & $0.088_{-0.04}^{3.00}$ & $0.05_{-0.02}^{29.00}$ \\
\hline
\end{tabular}

Notes. For each line, Gaussian FWHM $(\AA)$, line positions $(\AA)$ and intensities $\left(\mathrm{ct} \mathrm{s}^{-1} \AA^{-1}\right)$ are indicated. 